\section{Analyses of the FranceCoag cohort support differences in immunogenicity among one plasma-derived and two recombinant factor VIII brands in boys with severe hemophilia A}

\author{
Thierry Calvez, ${ }^{1}$ Hervé Chambost, ${ }^{2,3}$ Roseline d'Oiron, ${ }^{4}$ Vincent Dalibard, ${ }^{5}$ \\ Virginie Demiguel, ${ }^{6}$ Alexandra Doncarli, ${ }^{6}$ Yves Gruel, ${ }^{7}$ Yoann Huguenin, ${ }^{8}$ \\ Patrice Lutz, ${ }^{9}$ Chantal Rothschild, ${ }^{10}$ Christine Vinciguerra ${ }^{11}$ and Jenny \\ Goudemand $^{5,12}$ for FranceCoag Collaborators*
}

${ }^{1}$ Sorbonne Universités, UPMC Paris 06, Inserm, Institut Pierre Louis d'Épidémiologie et de Santé Publique (IPLESP UMRS 1136); ${ }^{2}$ Service d'Hématologie Oncologie Pédiatrique, La Timone, APHM, Marseille; ${ }^{3}$ Aix Marseille University, INSERM, INRA, NORT, Marseille; ${ }^{4}$ Centre Régional de Traitement de l'Hémophilie, Hôpital Bicêtre, Hôpitaux Universitaires Paris Sud, APHP, Le Kremlin Bicêtre; ${ }^{5}$ Département d'Hématologie et de Transfusion, Centre Hospitalier Universitaire de Lille, Université Lille 2, EA 2693, Faculté de Médecine; ${ }^{6}$ Santé Publique France, French National Public Health Agency, SaintMaurice; 'Centre Régional de Traitement de I'Hémophilie, Laboratoire d'Hématologie, UMR CNRS 7292, Université François Rabelais, Tours; ${ }^{8}$ Service d'Hématologie, Hôpital Pellegrin Tripode, Bordeaux; 'Unité Pédiatrique d'Hématologie Oncologie, Hốpital Hautepierre, Strasbourg: ${ }^{10}$ Centre Régional de Traitement de I'Hémophilie, Hôpital Necker, APHP, Paris; ${ }^{11}$ Service d'Hématologie Biologique, Hospices Civils de Lyon, EA 4609, Université de Lyon and ${ }^{12}$ Institut Pasteur de Lille, EGID, Inserm UMR 1011, Université Lille 2, France

*FranceCoag Collaborators ( $n=111$ ) Adjaoud D, Aouba A, Ardillon L, Barbay V, Barro C, Bastenaire $B$, Bayart S, Behar C, Benz-Lemoine E, Berger C, Berny K, Bertrand MA, Beurrier P, Bianchin M, Biernat J, Biron-Andreani C, Blanc L, Borg JY, Bovet J, Briquel ME, Castet S, Coatmelec B, Codine $P$, Costa C, Costagliola D, De Lumley L, De Raucourt E, Demay Y, Derlon A, Desprez D, Deville A, Donadel Claeyssens S, Donadio D, Dumesnil C, Durin-Assollant A, Dutrillaux F, Falaise C, Faradji A, Ferry N, Fiks Sigaud M, Fimbel B, Fouassier M, Fressinaud E, Frotscher B, Gaillard S, Gautier P, Gay V, Gembara P, Gorde S, Grémy I, Guerois C, Guillaume Y, Guillet B, Guérin V, Harroche A, Hassoun A, Henni T, Lambert T, Laurian Y, Legrand F, Li-Thiao-Te V, Lienhart A, Macchi L, Meunier S, Micheau M, Milien V, Monlibert B, Monpoux F, Moreau P, Munzer M, Navarro R, Négrier C, Normand C, Nyombe P, Oudot C, Ounnoughene N, Pan Petesch B, Parquet A, Paugy P, Pautard B, Peynet J, Pincemaille $O$, Pineau-Vincent $F$, Polack B, Pouille Lievin O, Pouplard C, Pouzol P, Rafowicz A, Rauch A, Regina S, Ricard C, Robert V, Rospide P, Ryman A, Sainte Marie I, Sannié T, Schneider P, Schoepfer C, Schved JF, Stieltjes N, Stoven C, Tarral E, Thiercelin Legrand MF, Tintillier V, Toguyeni E, Torchet MF, Trossaërt M, Valentin JB, Vannier JP, Volot F, Wibaut B

\section{ABSTRACT}

A round one third of boys with severe hemophilia A develop inhibitors (neutralizing antibodies) against their therapeutic factor VIII product. This adverse effect may result in more lifethreatening bleeding, disability, impaired quality of life, and costly care. We compared the incidence of inhibitors in boys treated with the three factor VIII products most used in France: one plasma-derived (Factane) and two recombinant products (Advate and Kogenate Bayer). A previously untreated cohort of patients was created in 1994 to investigate risk factors for inhibitor development. We selected boys with severe hemophilia A (factor VIII $<1 \mathrm{IU} / \mathrm{dL}$ ) first treated with one of the three factor VIII products studied. Details of product infusions, inhibitor assays and main fixed and time-varying inhibitor risk factors were recorded for the first 75 exposure days. Three outcomes (all inhibitors, high-titer inhibitors and subsequently treated inhibitors) were analyzed by univariate and multivariate Cox models. We studied 395 boys first treated between 2001 and 2016 (131, 137, and 127 with Factane, Advate, and Kogenate Bayer, respectively). Clinically significant inhibitors were diagnosed in 121 patients (70 high-titer). The incidence of high-titer inhibitors was significantly associated with the factor VIII product received $(P=0.005)$ : the cumulative incidence at 75 exposure days was 12.7\% (95\% CI: 7.7-20.6) with Factane, 20.4\% (95\% CI: 14.0-29.1) with
Haematologica 2018

Volume 103(1):179-189

\section{Correspondence:}

thierry.calvez@iplesp.upmc.fr

Received: June 16, 2017.

Accepted: October 5, 2017.

Pre-published: October 12, 2017.

doi:10.3324/haematol.2017.174706

Check the online version for the most updated information on this article, online supplements, and information on authorship \& disclosures: www.haematologica.org/content/103/1/179

\section{(C)2018 Ferrata Storti Foundation}

Material published in Haematologica is covered by copyright. All rights are reserved to the Ferrata Storti Foundation. Use of published material is allowed under the following terms and conditions:

https://creativecommons.org/licenses/by-nc/4.0/legalcode. Copies of published material are allowed for personal or internal use. Sharing published material for non-commercial purposes is subject to the following conditions:

https://creativecommons.org/licenses/by-nc/4.0/legalcode, sect. 3. Reproducing and sharing published material for commercial purposes is not allowed without permission in writing from the publisher. 
Advate, and 31.6\% (95\% CI: 23.5-41.7) with Kogenate Bayer. The low inhibitor incidence observed with Factane is concordant with recent findings from the SIPPET randomized trial. These consistent results from observational and experimental studies should lead to improved care for previously untreated patients and cost savings for healthcare systems worldwide.

\section{Introduction}

Hemophilia $\mathrm{A}$ is a hereditary disorder caused by a quantitative and/or qualitative deficiency of a coagulation protein, factor VIII (FVIII). Prevention and treatment of bleeding rely on repeated infusions of the deficient clotting factor. ${ }^{1}$ The first FVIII products were plasma-derived. From 1993 to 2012, six recombinant products were authorized in the European Union. This group of products has become the most prescribed in Western countries. ${ }^{2}$ Since the effective prevention of transmission of human immunodeficiency virus and hepatitis C virus infections via blood products in the late 1980s, inhibitors (neutralizing antibodies against therapeutic FVIII) have been the most serious adverse effect of the treatment of hemophilia A. Around one third of boys with severe hemophilia A (FVIII $<0.01 \mathrm{IU} / \mathrm{dL}$ ) develop inhibitors during their first 50 exposure days (EDs). An ED is defined as a day when at least one FVIII dose is infused. In most cases, inhibitors substantially impair the outcome of hemophilia A and increase its management cost. ${ }^{3}$ The identification of modifiable inhibitor risk factors (FVIII product or other treatment modalities) is therefore a major issue. Unfortunately, to date, firm knowledge is lacking, leaving regulatory authorities, prescribers and hemophilia patients in doubt. Most knowledge comes from observational studies, of varying quality, which compare treatment modalities in previously untreated patients (PUPs). In the 2000s, the first comparative studies, albeit limited, suggested that incidence of inhibitors was higher with recombinant products than with plasmaderived ones in PUPs with severe hemophilia $\mathrm{A}^{4-6}$ (hereafter, inhibitor risk associated with FVIII products is termed "immunogenicity" regardless of the mechanisms involved). In the 2010s, two systematic reviews ${ }^{7,8}$ and a patient-level meta-analysis ${ }^{9}$ did not support a potential difference in immunogenicity between recombinant and plasma-derived products in PUPs. In 2013, the largest and most extensive international PUP cohort study found no such difference, however, only 88 PUPs were treated with 15 different plasma-derived products. ${ }^{10}$ Unexpectedly, this study showed an inhibitor incidence difference between the two most prescribed recombinant products. In 2014, this difference was confirmed by ad hoc analyses of two national PUP cohorts. ${ }^{11,12}$ However, in the absence of demonstrated pathophysiological mechanisms, these results have been hotly debated. ${ }^{13-17}$ Nevertheless, they support the concept of considering the immunogenicity of each FVIII product rather than its source (recombinant versus plasma-derived). Launched in 2010 and published in 2016, the SIPPET trial focused on immunogenicity according to product source, demonstrating a higher incidence of inhibitors in children treated with recombinant products. ${ }^{18,19}$ Until now, SIPPET remains the only randomized trial addressing product immunogenicity in children with hemophilia A. Such trials are challenging as the target population is very young, and the children often require immediate treatment at diagnosis. Thus regulatory agencies and authors have recommended systematic enrollment of PUPs in standardized national or international follow-up to rapidly determine the immunogenicity of newly marketed FVIII products. ${ }^{20-22}$ However, establishing such pharmacosurveillance systems takes time and currently very few well-documented PUP cohorts are available worldwide. In 1994, a national PUP cohort dedicated to the study of genetic and non-genetic inhibitor risk factors was established in France, ${ }^{23}$ where a single plasma-derived product has been overwhelmingly used since 2001. In this context, we compared the inhibitor incidence in PUPs with severe hemophilia A treated with this plasma-derived product and those treated with two recombinant products during the same period.

\section{Methods}

\section{Study design}

In France, the public health authorities created a national pharmacosurveillance system in 1994 for FVIII and factor IX products administered to hemophiliacs. ${ }^{23}$ Clinicians of all hemophilia treatment centers were invited to include all hemophilia patients in an observational open cohort. In 2003, this system was renamed FranceCoag and inclusion was extended to other hereditary bleeding disorders. The high observed average prevalence of hemophilia A at birth (23.3 cases per 100000 male live births for 1991-2008) compared with prevalences in other industrialized countries supports the exhaustiveness of this registry. ${ }^{11,24}$ Since 1994, PUPs with hemophilia (FVIII or factor IX $<2$ IU/dL) have been enrolled in a sub-cohort with detailed follow-up and data collection to investigate risk factors for inhibitor development and the impact of prophylaxis. FranceCoag is fully publicly funded, and governed by a steering committee representing all stakeholders (Online Supplementary Data). FranceCoag was authorized by the French data protection authority. Parents or legal guardians of PUPs were informed about its objectives and modalities and approved their child's enrollment in accordance with the Declaration of Helsinki.

\section{Patients}

From 2000 onward, all children diagnosed with hemophilia A and FVIII $<2$ IU/dL have had to be included in the FranceCoag PUP cohort before treatment initiation, or shortly thereafter. For this analysis, we selected boys with severe hemophilia A and welldocumented FVIII treatment with details of the first EDs (see the following paragraph). Among them, we selected boys first treated with a currently marketed product used sufficiently to enable informative comparisons.

\section{Data collection}

The data collected have been described previously. ${ }^{11,16}$ Briefly, in the FranceCoag PUP cohort, main fixed inhibitor risk factors (e.g., 
F8 gene defect, family history of hemophilia and inhibitor, ethnic origin) were recorded at inclusion or shortly thereafter. Quarterly visits were recommended until ED-150. At each follow-up visit, hemorrhagic events, surgical procedures, treatments received and results of all inhibitor assays since the previous visit were accurately recorded. All data were centralized via a dedicated website. In parallel, details of the first $75 \mathrm{EDs}$ [date, reason(s) for treatment, FVIII product, dose and body weight] were recorded on a spreadsheet for each patient from his own booklet and hospital records. Data were automatically checked for inconsistencies and closely monitored by three dedicated clinical research assistants who compared the database with the original files in the centers.

\section{Follow-up and outcomes}

The cutoff date was December 6, 2016. Only the first 75 EDs were considered. If inhibitors developed during this observational period, EDs were counted until the last ED before their detection.
If the patient had not reached $75 \mathrm{EDs}$ at the last clinical visit or in the event of death or a switch to another FVIII product, follow-up was censored after the last recorded ED with the initial product. Classic outcomes were considered: all clinically significant inhibitors defined as a positive result (titer $>0.6$ Bethesda units) found in two distinct samples and high-titer inhibitors with a peak titer of at least 5 Bethesda units at any time. As previously published, ${ }^{11}$ we also considered inhibitors subsequently treated at any time with a bypassing agent and/or immune tolerance induction, as these treatments impair the patient's quality of life and represent a significant economic burden for the community. Inhibitor assays were performed in each center's laboratory. All of these laboratories participate in the international external quality assessment program of the European Concerted Action on Thrombosis Foundation. ${ }^{25}$ All inhibitor cases were validated by an ad hoc committee using a standardized procedure (Online Supplementary Data).

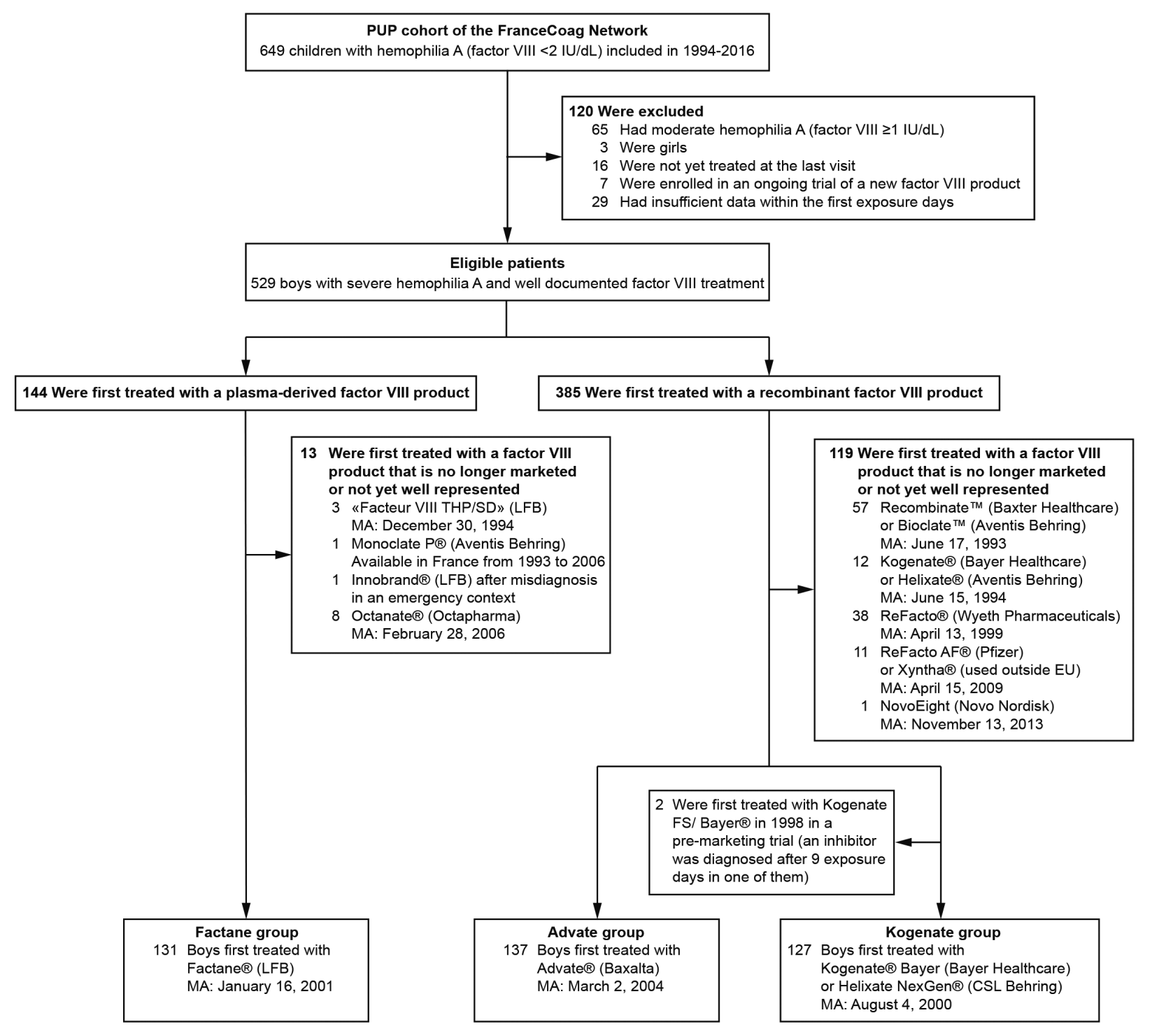

Figure 1. Patient selection process. At the cutoff date (December 6, 2016), 649 previously untreated patients (PUPS) with hemophilia A (factor VIII <2 IU/dL) had been included in the dedicated cohort of FranceCoag. After the selection process, three groups of boys with severe hemophilia A (factor VIII <1 IU/dL) were formed based on the first factor VIII product received. MA: marketing authorization dates in European Union (or in France for Factane ${ }^{\oplus}$ ). 


\section{Factor VIII products studied}

We compared the three FVIII products still marketed in the European Union and sufficiently represented in our PUP cohort (Figure 1). Factane ${ }^{\circledast}$ (manufactured by LFB) is a plasma-derived FVIII product that originates from cryoprecipitate of large pools of plasma from blood donors. It is co-purified by ion exchange chromatography with von Willebrand factor (20-40 IU per 100 IU FVIII). Its virus removal/inactivation steps include solvent/detergent inactivation and nanofiltration on 35 and 15 nm filters. ${ }^{26}$ Advate $^{\circledast}$ (Baxalta, acquired by Shire in 2016) is a third-generation recombinant FVIII product. The full-length FVIII protein is produced in Chinese hamster ovary cells. Neither human nor animal protein is used in the fermentation process and the lyophilized preparation is stabilized with trehalose and mannitol. ${ }^{27}$ Kogenate ${ }^{\circledR}$ Bayer (Bayer HealthCare) is a second-generation recombinant FVIII product. The full-length FVIII protein is produced in baby hamster kidney cells and the final preparation is stabilized with sucrose. ${ }^{28}$ This FVIII product is also distributed as Helixate ${ }^{\circledR}$ NexGen by CSL Behring. Hereafter, these three studied FVIII products are termed Factane, Advate, and Kogenate, respectively.

\section{Statistical analysis}

We compared inhibitor incidence between PUPs treated with the plasma-derived product widely used in France since 2001 (Factane) and those treated with the two recombinant products most used in the same period (Advate and Kogenate). First, the cumulative incidences of inhibitor according to product received were represented by Kaplan-Meier curves for the three outcomes. This representation was also used to analyze the product interactions with: the first exposure period, and treatment intensity at first exposure. Associations between the products received and inhibitor incidence were analyzed by a Cox proportional hazards model. Time was measured in terms of EDs, as is usual for inhibitor risk analyses. An ED was defined as a day during which one or more infusions of FVIII were given. As in our previous article ${ }^{11}$ in the case of a product switch, patients were considered as still being exposed to the first FVIII product for seven calendar days following the first infusion of the new product. This period was considered as the shortest latency between a risk factor exposure and a detectable related inhibitor occurrence. For the high-titer inhibitor analysis, the follow-up of patients who developed a low-titer inhibitor was censored at its onset. Similarly, for the analysis of treated inhibitors (with a bypassing agent and/or immune tolerance induction), the follow-up of patients who developed an untreated inhibitor was censored at its onset. Multivariate analysis included, individually and then together, four fixed cofactors (F8 gene defect, family history of hemophilia and inhibitor, ethnic origin, and age at first exposure) and five timevarying cofactors (calendar period, peak treatment episodes $\geq 5$ and $\geq 10$ consecutive EDs, severe bleeding episodes, and regular prophylaxis). Follow-up was censored upon any switch of product, so this factor was considered as fixed and Factane was chosen as the reference. The analyses were repeated, taking propensity scores into account by two methods: stratifying by quintiles of the propensity score and inverse probability of treatment weighting. ${ }^{29-31}$ Crude and adjusted hazard ratios with 95\% confidence intervals (95\% CI) were reported for Advate versus Factane and then for Kogenate versus Factane. Stata statistical software release 13.1 (College Station, TX StataCorp LP, USA) was used for all analyses and figures. For cofactor definitions, grouping, missing data procedures, and propensity score analyses, see the Online Supplementary Methods.

\section{Sensitivity analyses}

Two sensitivity analyses were performed, one by selecting patients first treated in the same period (from 2004 for Advate versus Factane comparisons, and until 2013 for Kogenate versus Factane comparisons) and the other by using real time instead of $\mathrm{ED}$ as the time unit in Cox models.

\section{Results}

\section{Selection and characteristics of the patients}

In all, 649 children with hemophilia A were included by 35 centers in the FranceCoag PUP cohort between 1994 and 2016. For this analysis, 120 ineligible patients were excluded (Figure 1). The 29 patients excluded due to insufficient data were first treated with nine well-balanced FVIII products. An inhibitor was diagnosed before ED 75 in five of them (first treated with five different FVIII products). Among the remaining 529 patients, 144 were first treated with plasma-derived FVIII products and 385 with recombinant products. We analyzed three product groups (Factane, Advate and Kogenate) which were sufficiently large to provide informative comparisons. Calendar period being a potential confounder, we excluded two patients first treated with Kogenate 2 years before its marketing authorization. Finally, 395 patients first treated with Factane, Advate and Kogenate $(n=131$, 137 and 127, respectively) between 2001 and 2016 were included in our analyses. Baseline characteristics and time-varying cofactors according to the FVIII product received are presented in Table 1 . No significant association between product and cofactors was observed, except for calendar period of first exposure to FVIII $(P<0.001)$ and $F 8$ gene defect $(P=0.009)$. Advate was marketed more than 3 years after Factane and Kogenate; initial treatment with Kogenate decreased during 20132016 , probably due to consistent results published in $2013^{10}$ and $2014^{11,12}$ (Online Supplementary Table S1). Most PUPs with a not yet tested F8 gene defect (14 out of 18) were born in 2013 or after and were, therefore, treated with Factane or Advate. Without the undetermined F8 gene defect modality, no significant imbalance between product groups was observed $(P=0.226)$.

\section{Follow-up and exposure to factor VIII}

Overall 18,244 EDs were recorded during 559.5 personyears (Online Supplementary Table S2). Among the 274 patients without inhibitors, 194 (70.8\%) were followed up until ED 75. Among the 80 patients with a censored follow-up before ED 75, two died, 24 switched to another FVIII product (see details in Online Supplementary Table S3) and 54 had not reached ED 75 at the last clinical visit. The contributions in EDs according to the FVIII product received and the studied time-varying risk factors are shown in Online Supplementary Table S4.

\section{Inhibitor assay frequency and inhibitor incidence}

Altogether, 2,002 inhibitor assays were documented for the three groups of patients during the observation period. On average, these assays were performed every 6.0 EDs during the first $25 \mathrm{EDs}$ and every 9.1 EDs during the overall follow-up period. The assay frequency was similar across the FVIII product groups (Online Supplementary Table S5). A clinically significant inhibitor was diagnosed in 121 patients after a median of 14 EDs (interquartile range, 8-20 EDs) and 
at a median age of 16.0 months (interquartile range, 12.024.0 months) (Table 2). Among them, 70 (57.9\%) had hightiter inhibitors and $104(86.0 \%)$ were subsequently treated with a bypassing agent and/or immune tolerance induction at some time during the entire FranceCoag follow-up. The global cumulative incidence at 75 EDs was $35.0 \%$ (95\% CI: $30.2 \%-40.3 \%$ ) for all inhibitors, $21.3 \%$ (95\% CI: $17.2 \%-$ $26.2 \%$ ) for high-titer inhibitors and 30.5\% (95\% CI: $25.9 \%$ $35.7 \%$ ) for subsequently treated inhibitors.
Inhibitor incidence according to factor VIII product

Inhibitor incidence was significantly associated with the product received for all inhibitors $(P<0.001)$, high-titer inhibitors $(P=0.005)$ and treated inhibitors $(P<0.001)$ (Figure 2A). For high-titer inhibitors, the cumulative incidence at 75 EDs was $12.7 \%$ (95\% CI: 7.7-20.6) with Factane, 20.4\% (95\% CI: 14.0-29.1) with Advate, and 31.6\% (95\% CI: 23.541.7) with Kogenate (Table 3). Results were similar in weighted analyses using propensity scores (Figure 2B).

Table 1. Patients' characteristics according to the factor VIII product received.

\begin{tabular}{|c|c|c|c|c|}
\hline Fixed risk factors & $\begin{array}{l}\text { Factane } \\
(\mathrm{N}=131)\end{array}$ & $\begin{array}{c}\text { Advate } \\
(\mathrm{N}=137)\end{array}$ & $\begin{array}{l}\text { Kogenate } \\
\text { (N = 127) }\end{array}$ & $\boldsymbol{P}$ \\
\hline $\begin{array}{l}\text { F8 gene defect — no. (\%) } \\
\text { Low risk } \\
\text { High risk* } \\
\text { Undetermined (e.g., not yet tested, unidentified) }\end{array}$ & $\begin{array}{cc}37 & (28.2) \\
83 & (63.4) \\
11 & (8.4)\end{array}$ & $\begin{array}{cc}33 & (24.1) \\
97 & (70.8) \\
7 & (5.1)\end{array}$ & $\begin{array}{c}27(21.3) \\
100(78.7) \\
0 \quad(0.0)\end{array}$ & 0.009 \\
\hline High-risk F8 gene defect known at first FVIII infusion & 31 (23.7) & $40(29.2)$ & $35(27.6)$ & 0.579 \\
\hline $\begin{array}{l}\text { Family history }-\mathrm{n} .(\%) \\
\text { Hemophilia without inhibitor } \\
\text { Hemophilia with inhibitor } \\
\text { No family history of hemophilia }\end{array}$ & $\begin{array}{cc}47 & (35.9) \\
7 & (5.3) \\
77 & (58.8)\end{array}$ & $\begin{array}{ll}57 & (41.6) \\
13 & (9.5) \\
67 & (48.9)\end{array}$ & $\begin{array}{ll}38 & (29.9) \\
15 & (11.8) \\
74 & (58.3)\end{array}$ & 0.128 \\
\hline Family history of hemophilia and inhibitor known at first FVIII infusion & $3 \quad(2.3)$ & $9 \quad(6.6)$ & $6 \quad(4.7)$ & 0.243 \\
\hline $\begin{array}{l}\text { Ethnic origin - n. }(\%)^{\dagger} \\
\text { White only (both parents) } \\
\text { Other, not African or Afro-American } \\
\text { African or Afro-American (at least one grandparent) }\end{array}$ & $\begin{array}{ll}91 & (69.5) \\
27 & (20.6) \\
13 & (9.9)\end{array}$ & $\begin{array}{cc}100 & (73.0) \\
25 & (18.2) \\
12 & (8.8)\end{array}$ & $\begin{array}{cc}93 & (73.2) \\
29 & (22.8) \\
5 & (3.9)\end{array}$ & 0.372 \\
\hline $\begin{array}{l}\text { Calendar period of first exposure to FVIII — n. (\%) } \\
2001-2003 \\
2004-2006 \\
2007-2009 \\
2010-2012 \\
2013-2016\end{array}$ & $\begin{array}{ll}21 & (16.0) \\
16 & (12.2) \\
37 & (28.2) \\
18 & (13.7) \\
39 & (29.8)\end{array}$ & $\begin{array}{cc}0 & (0.0) \\
35 & (25.5) \\
36 & (26.3) \\
31 & (22.6) \\
35 & (25.5)\end{array}$ & $\begin{array}{cc}34 & (26.8) \\
22 & (17.3) \\
35 & (27.6) \\
31 & (24.4) \\
5 & (3.9)\end{array}$ & $<0.001$ \\
\hline $\begin{array}{l}\text { Age at first exposure to FVIII - n. (\%) } \\
\text { Less than } 6 \text { months } \\
6 \text {-11 months } \\
\text { At least } 12 \text { months }\end{array}$ & $\begin{array}{ll}44 & (33.6) \\
41 & (31.3) \\
46 & (35.1)\end{array}$ & $\begin{array}{ll}39 & (28.5) \\
54 & (39.4) \\
44 & (32.1)\end{array}$ & $\begin{array}{ll}26 & (20.5) \\
43 & (33.9) \\
58 & (45.7)\end{array}$ & 0.063 \\
\hline
\end{tabular}

\section{Risk factors at first exposure (fixed risk factors)} Peak treatment episode at first exposure — n. (\%)

At least 3 consecutive EDs

At least 5 consecutive EDs

At least 10 consecutive EDs

$38(29.0)$

$26(19.8)$

$14(10.7)$

First exposure linked to surgical procedure (with at least 3 EDs) - n. (\%)

First exposure linked to severe bleeding episode - n. (\%)
$7 \quad(5.3)$

$11(8.4)$

$\begin{array}{cc}40 & (29.2) \\ 20 & (14.6) \\ 10 & (7.3) \\ 6 & (4.4) \\ 14 & (10.2)\end{array}$

37 (29.1)

\begin{tabular}{|c|c|c|c|c|}
\hline $\begin{array}{l}\text { History of peak treatment episodes ( } \geq 1 \text { during follow-up) - n. (\%) } \\
\geq 3 \text { consecutive EDs } \\
\geq 5 \text { consecutive EDs } \\
\geq 10 \text { consecutive EDs }\end{array}$ & $\begin{array}{ll}86 & (65.6) \\
56 & (42.7) \\
21 & (16.0)\end{array}$ & $\begin{array}{ll}90 & (65.7) \\
50 & (36.5) \\
18 & (13.1)\end{array}$ & $\begin{array}{ll}83 & (65.4) \\
46 & (36.2) \\
18 & (14.2)\end{array}$ & $\begin{array}{l}0.998 \\
0.470 \\
0.793\end{array}$ \\
\hline $\begin{array}{l}\text { History of surgical procedures (with } \geq 3 \text { EDs) during follow-up - n. (\%) } \\
\text { History of severe bleeding episodes during follow-up - n. (\%) }\end{array}$ & $\begin{array}{ll}21 & (16.0) \\
14 & (10.7)\end{array}$ & $\begin{array}{ll}19 & (13.9) \\
17 & (12.4)\end{array}$ & $\begin{array}{lc}12 & (9.4) \\
18 & (14.2)\end{array}$ & $\begin{array}{l}0.282 \\
0.697\end{array}$ \\
\hline $\begin{array}{l}\text { Initiation of regular prophylaxis } \\
\text { N. (\% according to Kaplan-Meier estimator) at } 25 \text { EDs } \\
\text { N. (\% according to Kaplan-Meier estimator) at } 50 \text { EDs }\end{array}$ & $\begin{array}{ll}51 & (65.4) \\
73 & (93.6)\end{array}$ & $\begin{array}{ll}63 & (74.1) \\
80 & (94.1)\end{array}$ & $\begin{array}{l}40(60.6) \\
61(92.4)\end{array}$ & $0.153 \S$ \\
\hline
\end{tabular}

${ }^{*}$ High-risk $F 8$ gene defects include large deletions (at least 1 exon), intron 1 and 22 inversions, small deletions/insertions with stop codon (out of A-run) and nonsense mutations. 'Up to four ethnic origins per patient could be recorded (one for each grandparent). "The initiation of regular prophylaxis was defined as the moment at which at least three consecutive prophylactic infusions of FVIII were given within a period of at least 15 days (RODIN definition). ${ }^{10}{ }^{~} \mathrm{P}$ for global test using Cox proportional hazards model with exposure day as the observational time unit. FVIII: factor VIII; ED: exposure day. 


\section{Interaction analyses}

Patients were split into two roughly equal groups according to calendar period of first exposure to FVIII. The immunogenicity differences across FVIII products were similar for both periods (Figure 3A) and the interactions between period and FVIII product were not statistically significant $(P \geq 0.444)$. Similarly, no significant interactions between peak treatment episode at first exposure and FVIII product were observed (Figure 3B).

\section{Advate versus Factane}

The crude hazard ratio of Advate versus Factane was

Table 2. Characteristics of clinically significant inhibitors.

\begin{tabular}{|c|c|c|c|c|c|}
\hline & \multicolumn{2}{|c|}{$\begin{array}{l}\text { All inhibitors } \\
(\mathrm{N}=121)\end{array}$} & $\begin{array}{l}\text { High-titer inhibitors* } \\
\qquad(\mathrm{N}=70)\end{array}$ & \multicolumn{2}{|c|}{$\begin{array}{l}\text { Low-titer } \\
\text { inhibitors } \\
(\mathbb{N}=51)\end{array}$} \\
\hline Median number of EDs at inhibitor detection (IQR) & 14 & $(8-20)$ & $(8-18)$ & 17 & $(10-24)$ \\
\hline Median age at inhibitor detection (IQR) — months & 16.0 & $(12.0-24.0)$ & $13.4(8.6-20.2)$ & 19.3 & $(15.1-28.5)$ \\
\hline Median duration between ED 1 and inhibitor detection (IQR) — months & 5.3 & $(1.9-10.1)$ & $3.0 \quad(1.2-7.4)$ & 7.3 & $(2.8-14.8)$ \\
\hline Median of maximal inhibitor titer (IQR) — Bethesda units & 8.0 & $(2.5-72.0)$ & $46.0(15.0-256.0)$ & 2.0 & $(1.1-3.3)$ \\
\hline
\end{tabular}

\section{Treatments received at any time during the FranceCoag follow-up}

\begin{tabular}{|c|c|c|c|c|c|c|}
\hline Treatment with bypassing agents — n. (\%) & 88 & $(72.7)$ & 64 & $(91.4)$ & 24 & $(47.1)$ \\
\hline Treatment with immune tolerance induction (ITI) - n. (\%) & 89 & $(73.6)$ & 62 & $(88.6)$ & 27 & $(52.9)$ \\
\hline Treatment with bypassing agents and/or ITI — n. (\%) & 104 & $(86.0)$ & 70 & $(100.0)$ & 34 & $(66.7)$ \\
\hline
\end{tabular}

* High-titer inhibitor defined as peak titer equal to or greater than 5 Bethesda units at any time during the FranceCoag follow-up. ED: exposure day; IQR: interquartile range

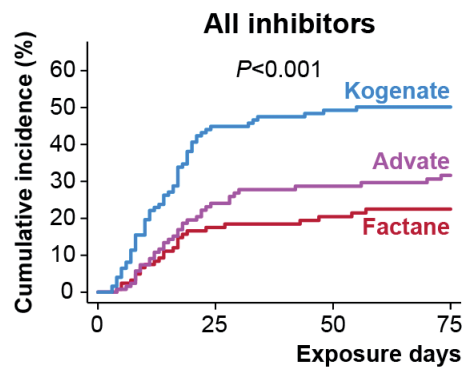

$\begin{array}{lllll}\text { No. at risk } & & & & \\ \text { Factane } & 131 & 89 & 79 & 69 \\ \text { Advate } & 137 & 82 & 76 & 69 \\ \text { Kogenate } & 127 & 64 & 57 & 56\end{array}$
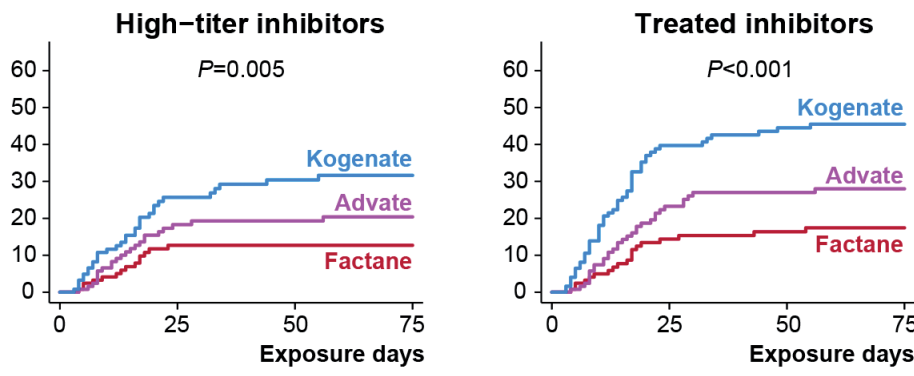

$\begin{array}{llll}131 & 89 & 79 & 69 \\ 137 & 82 & 76 & 69 \\ 127 & 64 & 57 & 56\end{array}$

$\begin{array}{llll}131 & 89 & 79 & 69 \\ 137 & 82 & 76 & 69 \\ 127 & 64 & 57 & 56\end{array}$

\section{B Weighted Kaplan-Meier estimates}
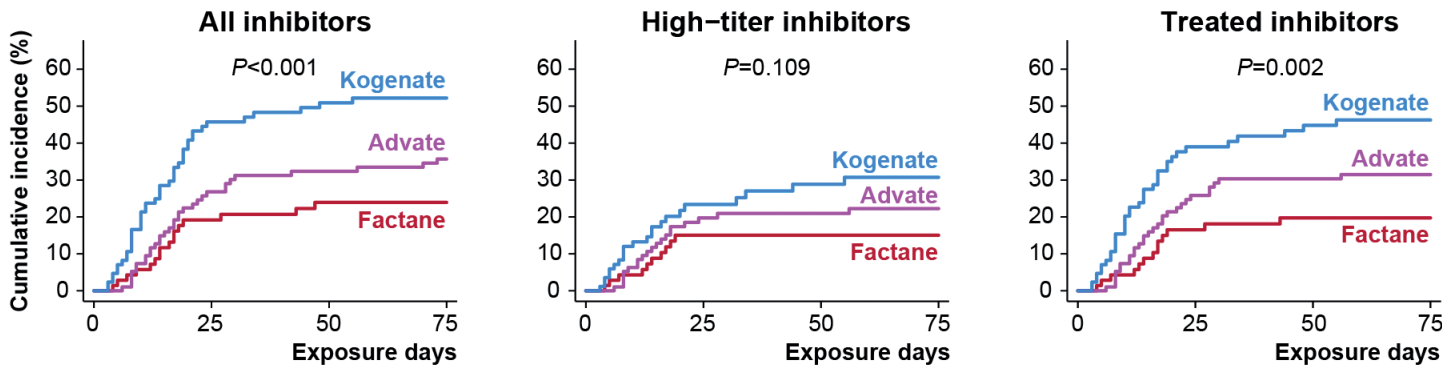

$\begin{array}{lrrrr}\text { No. at risk } & & & & \\ \text { Factane } & 71 & 53 & 46 & 42 \\ \text { Advate } & 102 & 66 & 61 & 58 \\ \text { Kogenate } & 88 & 43 & 38 & 37\end{array}$

$\begin{array}{rlll}71 & 53 & 46 & 42 \\ 102 & 66 & 61 & 58 \\ 88 & 43 & 38 & 37\end{array}$

Figure 2. Kaplan-Meier representation of the cumulative incidence of inhibitors, with exposure day as the observational time unit, according to the factor VIII product received. Three outcomes are shown: all inhibitors, high-titer inhibitors and inhibitors subsequently treated with a bypassing agent and/or immune tolerance induction. (A) Kaplan-Meier estimates are shown for all patients. Tests used Cox proportional hazards model. (B) Weighted Kaplan-Meier estimates are shown for patients first treated between 2004 and 2012. This selection was made to avoid having patients with an extremely low probability of having received one of the two counterfactual treatments (see Online Supplementary Methods). Tests used weighted Cox proportional hazards model. 
1.63 (95\% CI: 0.84-3.17) for high-titer inhibitors (Figure $4 \mathrm{~A}$ and Online Supplementary Table S7). Repeated analyses were adjusted first for each cofactor, then for all fixed and then for all time-varying cofactors, and showed comparable results. Finally, adjusted hazard ratios of Advate versus Factane were 1.64 (95\% CI: 0.823.25 ) in the complete model (including all fixed and time-varying cofactors) and 1.54 (95\% CI: 0.73-3.24) in the propensity score analysis using inverse probability of treatment weighting and adjustment for time-varying risk factors. Results were similar for the two sensitivity analyses and the two other outcomes: all inhibitors and treated inhibitors (Figure 4A, Online Supplementary Tables S6 and S8).

\section{Kogenate versus Factane}

The crude hazard ratio for Kogenate versus Factane was 2.68 (95\% CI: 1.43-5.00) for high-titer inhibitors (Figure 4B, Online Supplementary Table S7). Repeated analyses were adjusted first for each cofactor, then for all fixed and for all time-varying cofactors, with comparable results. The adjusted hazard ratios for Kogenate versus
Factane were 2.81 (95\% CI: 1.44-5.49) in the complete model and 2.13 (95\% CI: 1.02-4.46) in the propensity score analysis using inverse probability of treatment weighting and adjustment for time-varying risk factors. Results were also similar for the two sensitivity analyses and for the two other outcomes (Figure 4B, Online Supplementary Tables S6 and S8).

\section{Discussion}

Since 1994, PUPs with severe hemophilia A have been prospectively followed up in France, notably to evaluate the immunogenicity of FVIII products. Whatever the outcome considered, adjusted results showed a highly significant difference $(P \leq 0.005)$ in the incidence of inhibitors among the groups receiving the three most used products between 2001 and 2016. To our knowledge, this study is the first to compare inhibitor incidence among large groups receiving single FVIII products, including a plasmaderived FVIII product. Firstly, we found a higher risk of inhibitor development, approximately $50 \%$, in PUPs treat-

\section{A By calendar period of first exposure to factor VIII}
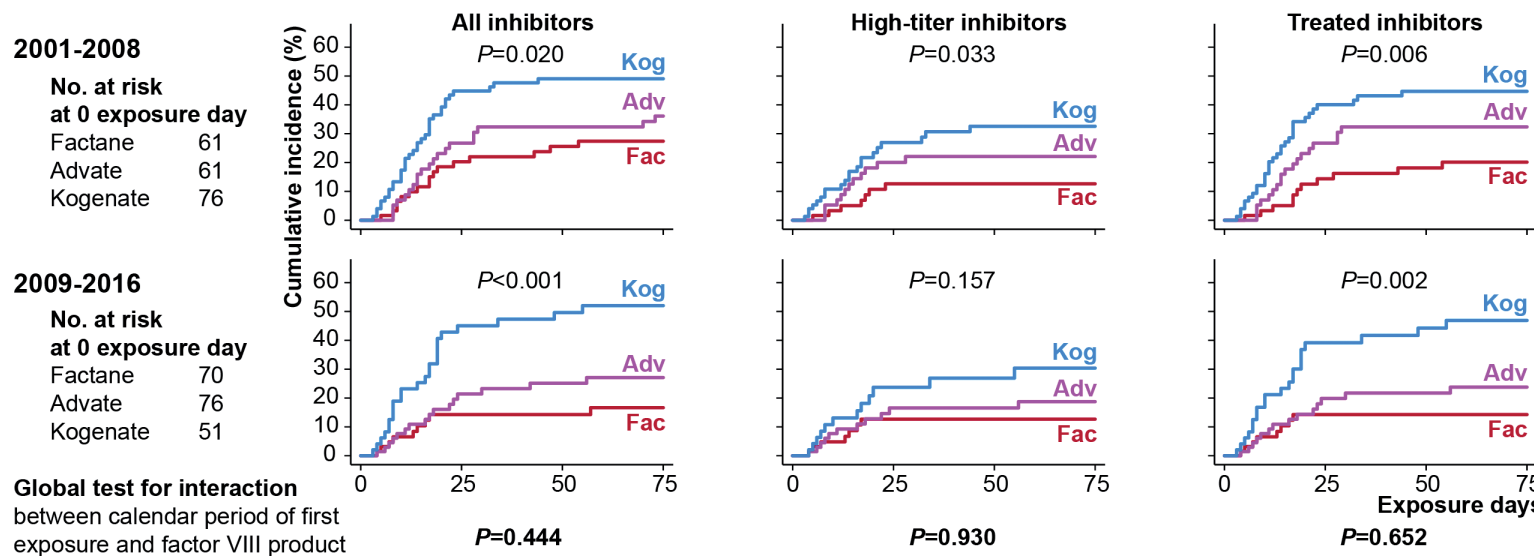

$P=0.444$

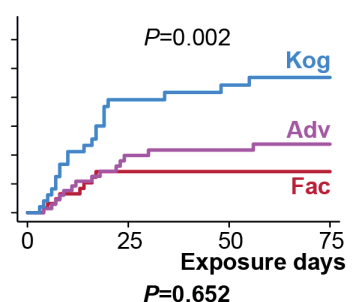

\section{B By peak treatment episode at first exposure}
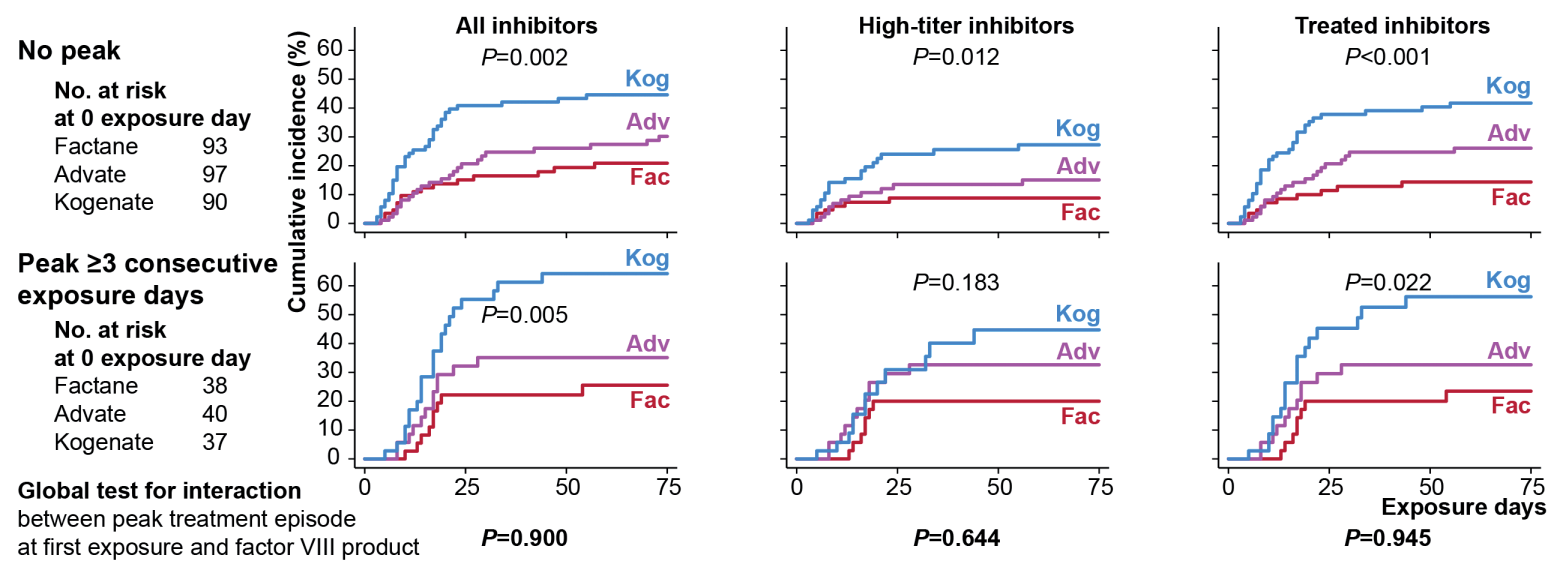

Figure 3. Kaplan-Meier representation of the cumulative incidence of inhibitors, with exposure day as the observational time unit, according to the factor VIII product received. Three outcomes are shown: all inhibitors, high-titer inhibitors and inhibitors subsequently treated with a bypassing agent and/or immune tolerance induction. Tests used Cox proportional hazards model. (A) Kaplan-Meier estimates according to calendar period of first exposure to factor VIII. (B) Kaplan-Meier estimates according to treatment intensity at first exposure (peak treatment episode $\geq 3$ consecutive exposure days). 
ed with Advate than in those treated with Factane. This result was stable across different models and sensitivity analyses, but not statistically significant for the three studied outcomes. However, the adjusted hazard ratio of
Advate versus Factane for high-titer inhibitors (1.64; 95\% CI: 0.82-3.25) was similar to that in the SIPPET trial in which the adjusted hazard ratio for recombinant FVIII versus plasma-derived FVIII was 1.69 (95\% CI: 0.96-2.98). ${ }^{32}$

\section{Advate versus Factane}

All inhibitors

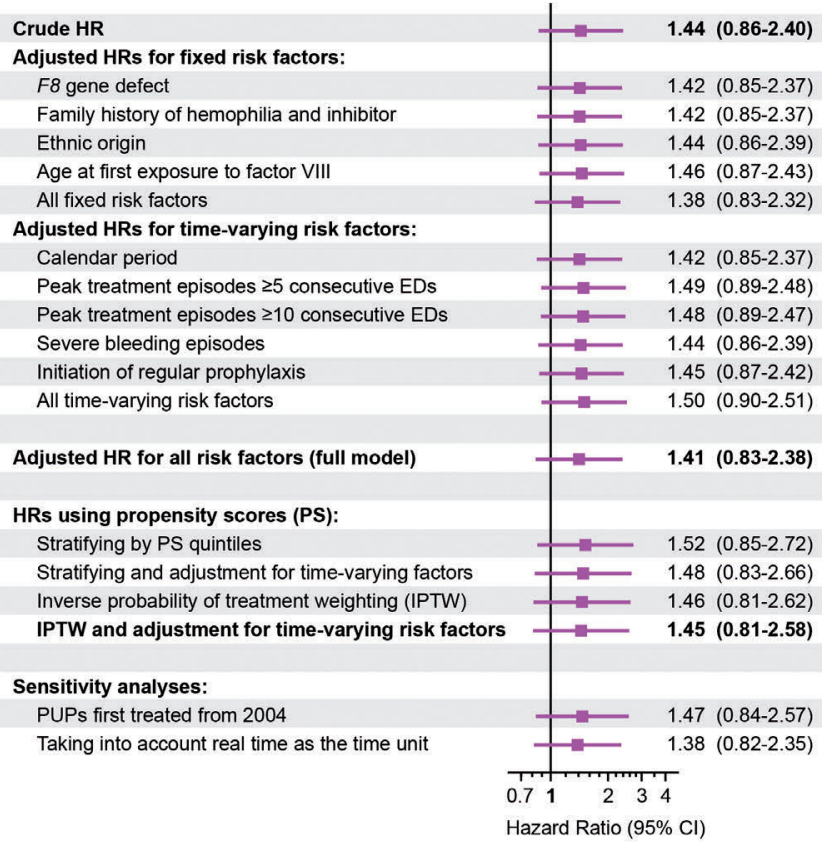

High-titer inhibitors

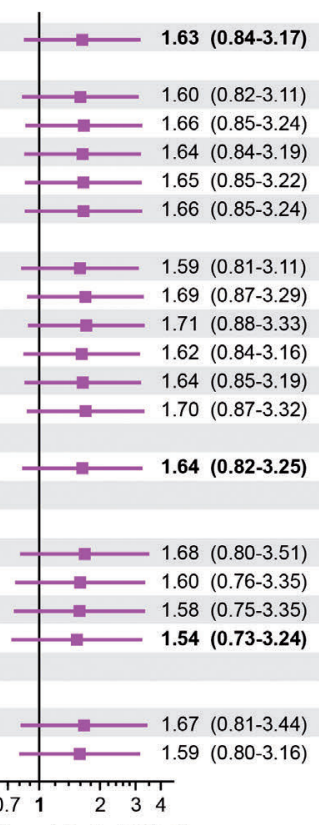

Hazard Ratio $(95 \% \mathrm{Cl})$
Treated inhibitors

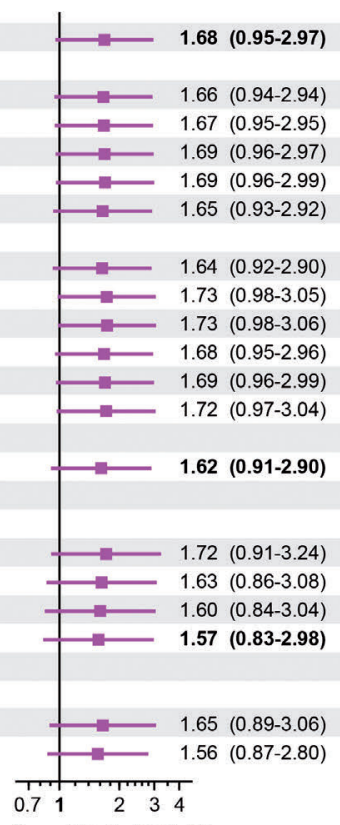

Hazard Ratio $(95 \% \mathrm{Cl})$

\section{B Kogenate versus Factane}

\begin{tabular}{|c|c|c|c|c|}
\hline \multirow[b]{2}{*}{ Crude HR } & \multicolumn{2}{|c|}{ All inhibitors } & High-titer inhibitors & Treated inhibitors \\
\hline & $\longrightarrow$ & $2.77(1.74-4.42)$ & $\longrightarrow-2.68(1.43-5.00)$ & $\longrightarrow-3.20(1.89-5.41)$ \\
\hline \multicolumn{5}{|l|}{ Adjusted HRs for fixed risk factors: } \\
\hline F8 gene defect & $\longrightarrow$ & $2.64(1.65-4.23)$ & $\longrightarrow-2.51(1.34-4.72)$ & $\longrightarrow-3.06(1.80-5.21)$ \\
\hline Family history of hemophilia and inhibitor & $\longrightarrow-$ & $2.64(1.65-4.23)$ & $\longrightarrow=2.61(1.39-4.90)$ & $\longrightarrow-3.07(1.81-5.21)$ \\
\hline Ethnic origin & $\rightarrow-$ & $2.85(1.78-4.55)$ & $\longrightarrow-2.73(1.46-5.11)$ & $\longrightarrow-3.30(1.95-5.57)$ \\
\hline Age at first exposure to factor VIII & $\longrightarrow-$ & $2.80(1.75-4.48)$ & $\longrightarrow-2.79(1.49-5.22)$ & $\longrightarrow-3.23(1.91-5.47)$ \\
\hline All fixed risk factors & $\longrightarrow$ & $2.58(1.60-4.16)$ & $\longrightarrow-2.64(1.39-5.00)$ & $\longrightarrow-3.02(1.76-5.17)$ \\
\hline \multicolumn{5}{|l|}{ Adjusted HRs for time-varying risk factors: } \\
\hline Calendar period & $\rightarrow-$ & $2.81(1.75-4.52)$ & $\longrightarrow-2.75(1.46-5.18)$ & $\longrightarrow-3.21(1.89-5.46)$ \\
\hline Peak treatment episodes $\geq 5$ consecutive EDs & $\longrightarrow-$ & $2.80(1.76-4.47)$ & $\longrightarrow-2.71(1.45-5.07)$ & $\longrightarrow-3.23(1.91-5.46)$ \\
\hline Peak treatment episodes $\geq 10$ consecutive EDs & $\longrightarrow$ & $2.80(1.75-4.47)$ & $\longrightarrow-2.68(1.43-5.01)$ & $\longrightarrow-3.22(1.91-5.44)$ \\
\hline Severe bleeding episodes & $\longrightarrow$ & $2.68(1.68-4.28)$ & $\longrightarrow-2.55(1.36-4.77)$ & $\longrightarrow-3.09(1.83-5.23)$ \\
\hline Initiation of regular prophylaxis & $\longrightarrow$ & $2.70(1.69-4.31)$ & $\longrightarrow=2.61(1.40-4.89)$ & $\longrightarrow-3.13(1.85-5.30)$ \\
\hline All time-varying risk factors & $\longrightarrow$ & $2.89(1.79-4.67)$ & $\longrightarrow-2.86(1.50-5.48)$ & $\longrightarrow-3.29(1.92-5.64)$ \\
\hline Adjusted HR for all risk factors (full model) & $\longrightarrow$ & $2.74(1.67-4.48)$ & $\longrightarrow-2.81(1.44-5.49)$ & $\longrightarrow-3.12(1.79-5.43)$ \\
\hline \multicolumn{5}{|l|}{ HRs using propensity scores (PS): } \\
\hline Stratifying by PS quintiles & $\longrightarrow$ & $2.40(1.41-4.08)$ & $\longrightarrow-2.32(1.14-4.74)$ & $\longrightarrow-2.66(1.46-4.82)$ \\
\hline Stratifying and adjustment for time-varying factors & $\longrightarrow$ & $2.51(1.46-4.33)$ & $\longrightarrow-2.45(1.19-5.03)$ & $\longrightarrow-2.74(1.50-5.02)$ \\
\hline Inverse probability of treatment weighting (IPTW) & $\longrightarrow$ & $2.26(1.32-3.88)$ & $\longrightarrow=2.11(1.01-4.38)$ & $\longrightarrow \quad 2.40(1.31-4.37)$ \\
\hline IPTW and adjustment for time-varying risk factors & $\longrightarrow$ & $2.20(1.28-3.77)$ & $\longrightarrow-2.13(1.02-4.46)$ & $\longrightarrow \quad 2.33(1.28-4.26)$ \\
\hline \multicolumn{5}{|l|}{ Sensitivity analyses: } \\
\hline PUPs first treated until 2013 & $\longrightarrow$ & $2.74(1.64-4.57)$ & $\longrightarrow-2.60(1.31-5.14)$ & $\longrightarrow-3.06(1.73-5.42)$ \\
\hline \multirow[t]{3}{*}{ Taking into account real time as the time unit } & $\longrightarrow$ & $2.82(1.72-4.61)$ & $\longrightarrow-2.83(1.45-5.51)$ & $\longrightarrow-3.10(1.78-5.38)$ \\
\hline & 2345 & & $\begin{array}{l}\text { † } \\
1\end{array} 23456$ & 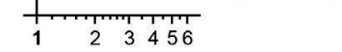 \\
\hline & ard Ratio $\left(95^{\circ}\right.$ & $\% \mathrm{Cl})$ & Hazard Ratio $(95 \% \mathrm{Cl})$ & Hazard Ratio (95\% Cl) \\
\hline
\end{tabular}


Hazard ratios were similar with propensity score analyses. Secondly, inhibitor incidence was at least twice as high in PUPs treated with Kogenate than in those treated with Factane. This result was stable across different analyses and highly significant for the three outcomes. Hazard ratios were slightly lower but still significant with propensity score analyses. This result was predictable considering the higher inhibitor incidence with Kogenate previously observed in our cohort, ${ }^{11}$ and in two other studies. ${ }^{10,12}$ We did not observe interactions between FVIII products and calendar periods or intensity of the initial FVIII treatment, unlike a recent study. ${ }^{9}$ However, our series was too small to study finer classifications. Interactions between other cofactors and FVIII products should be explored.

The higher inhibitor incidence in PUPs treated with Kogenate than in those treated with Advate reported in three independent observational studies sparked a lively debate. ${ }^{13,14}$ The main alleged bias has been confounding by indication. ${ }^{15}$ Clinicians might have preferentially prescribed Kogenate to patients most at risk of inhibitors after two publications highlighting its low immunogenicity. ${ }^{33,34}$ We showed that the absence of unidirectional imbalance in known risk factors at first FVIII infusion between product groups does not support this hypothesis. ${ }^{16}$ Some interviewed French clinicians acknowledged their willingness to use various brands of FVIII products in their center. Consequently, at least two of the studied FVIII products were used in most centers (Online Supplementary Table S9). However, the determinants in choosing a FVIII product for a given patient could be diverse, involving the views of the physician and/or family and also depending on environmental conditions. As data on such determinants were not collected, they cannot be precisely understood. Thus, residual confounding related to unknown or unregistered risk factors remains possible.

Since the 2000s, several studies have reported that plasma-derived products are less immunogenic in PUPs, especially those products with a high concentration of von Willebrand factor. ${ }^{4-6}$ A confounding by indication mechanism could therefore have led some French clinicians to treat most at-risk patients with Factane, inducing a higher incidence of inhibitors in this group. However, our results showed the opposite. Thus, confounding by indication related to known risk factors or subtle unrecorded patients' characteristics cannot explain the observed lower inhibitor incidence with Factane compared with Advate and Kogenate. Moreover, our results changed only slightly after integrating propensity scores to counter possible confounding by indication. This was consistent with the lack of systematic imbalance in risk factors between the groups receiving the different products. We consider a center-related bias is more conceivable. The relative proportions of PUPs treated with each FVIII product differed among centers. If centers have their own effect on inhibitor incidence, regardless of FVIII product and other considered cofactors, residual confounding would be possible. We integrated the size of the centers in our multivariate analyses without observing substantial variations in the results. As finally selected PUPs were distributed in 32 hemophilia treatment centers (1 to 53 PUPs per center), we could not integrate centers individually in our multivariate analyses. However, this bias is unlikely to explain the concordant results observed in the three observational studies conducted in independent areas. ${ }^{10-12}$ Moreover, it could not have affected the SIPPET trial. ${ }^{18,19}$
Table 3. Number of inhibitors and cumulative incidence at 75 exposure days according to the factor VIII product received.

\begin{tabular}{lcccc} 
Outcome & N. & $\begin{array}{c}\text { Cumulative incidence } \\
\text { at 75 exposure days } \\
\%\end{array}$ & $P^{*}$ \\
& & $95 \%$ Cl) & \\
All inhibitors & 25 & 22.5 & $(15.8-31.5)$ & $<0.001$ \\
$\begin{array}{l}\text { Factane } \\
\text { Advate }\end{array}$ & 36 & 31.6 & $(23.9-41.1)$ & \\
\hline Kogenate & 60 & 50.1 & $(41.6-59.4)$ & \\
\hline High-titer inhibitors & & & & 0.005 \\
Factane & 14 & 12.7 & $(7.7-20.6)$ & \\
Advate & 23 & 20.4 & $(14.0-29.1)$ & \\
\hline Kogenate & 33 & 31.6 & $(23.5-41.7)$ &
\end{tabular}

Inhibitors treated with bypassing agents and/or immune tolerance induction

\begin{tabular}{llll} 
Factane & 19 & 17.4 & $(11.5-26.0)$ \\
Advate & 32 & 28.0 & $(20.7-37.2)$ \\
\hline Kogenate & 53 & 45.5 & $(36.9-55.1)$ \\
\hline
\end{tabular}

${ }^{*} P$ for the global test using Cox proportional hazards model with exposure day as the observational time unit.

Observational studies cannot prove a difference in immunogenicity between FVIII products. However, consistent results from several independent and rigorously conducted observational studies ${ }^{10,12}$ support the immunogenicity difference between Advate and Kogenate. Concerning plasma-derived products, the results of the randomized SIPPET trial and our study are also consistent, although SIPPET compared products according to their source whereas we compared three manufactured FVIII products (received by $47 \%$ of the SIPPET patients). Moreover, although SIPPET was mainly performed in developing countries $76 \%$ of patients were enrolled in India, Egypt, or Iran), ${ }^{32}$ while our study reflects real life in a Western country, both conjointly support the reality of a difference in immunogenicity between plasma-derived and recombinant products. Assuming an immunogenicity difference between two FVIII products as similar as Advate and Kogenate, an immunogenicity difference between all products is highly likely, particularly between products as different as Factane and recombinant products. Numerous genetic and non-genetic factors have been shown to be associated with the inhibitor incidence in PUPs. ${ }^{35,36}$ Depending on the combination of these factors, inhibitor probability may vary widely (10 to $90 \%$ ). ${ }^{37,38}$ Given recent studies, ${ }^{10-12,18}$ the assumption that the nature of the product received affects the inhibitor incidence is increasingly credible. All FVIII products could potentially have their own immunogenicity levels, but extraordinary background noise generated by other cofactors could have prevented their identification until recently. The rarity of hemophilia $A$ and the inadequacy of institutional support for comprehensive data collection in many countries are also responsible for this non-recognition. No convincing pathophysiological hypothesis has yet attempted to explain a possible higher incidence of inhibitors with Kogenate. Conversely, many recently reviewed hypotheses exist to explain a possible low inhibitor incidence with plasma-derived products. ${ }^{39,40}$ However, identifying predominant explanatory mechanisms is crucial to prove the 
existence of this difference and to sustain the development of less immunogenic recombinant products.

After the unexpected observation of an immunogenicity difference between Advate and Kogenate in PUPs, some authors stressed that the primacy of randomized trials and the 5\% significance level (commonly required in drug efficacy assessment) were not relevant for considering drug adverse effects, a fortiori when alternative therapies exist. ${ }^{14}$ Thus, this result led to revised therapeutic recommendations. ${ }^{41}$ Oddly, although SIPPET has been the only randomized trial addressing an immunogenicity difference among FVIII products, yielding significant results, many experts advocated against broad changes in clinical practice. ${ }^{42-44}$ SIPPET and our results cannot be applied to patients with moderate/mild hemophilia A or severe hemophilia with over 50/100 EDs, when the inhibitor incidence is considerably lower (about 3 per 1000 person-years). ${ }^{45}$ However, when initiating replacement treatment in PUPs with severe hemophilia A, prescribers cannot ignore the risk/benefit of the different products, including imperfect knowledge of their immunogenicity. Moreover, at a time when several new recombinant FVIII products, including extended half-life ones, are entering the market with very little knowledge of their specific immunogenicity, it is crucial that all stakeholders organize accurate follow-up of all treated hemophilia A patients, and particularly PUPs with severe hemophilia A.

\section{Acknowledgments}

The authors thank Dominique Costagliola for critical review of the manuscript and helpful discussions. We also thank all the contributors from the FranceCoag Network, patients and their parents or legal guardians for their participation. TC thanks Jennifer Markovic for English editing assistance.

The French hemophilia surveillance system ("Suivi thérapeutique National des Hémophiles" since 1994 and "FranceCoag" from 2003 onward) is fully supported by the public health authorities. Additional data collection for the first 75 exposure days was partially supported by the French National Clinical Research Program (2009) and by Assistance Publique Hôpitaux de Marseille.

\section{References}

1. Peyvandi F, Garagiola I, Young G. The past and future of haemophilia: diagnosis, treatments, and its complications. Lancet. 2016:388(10040):187-197.

2. World Federation of Hemophilia. Report on the Annual Global Survey 2014 [Internet]. 2015 [cited Mar 17, 2016];Available from: http://www.wfh.org/en/data-collection

3. Di Minno MND, Di Minno G, Di Capua M, Cerbone AM, Coppola A. Cost of care of haemophilia with inhibitors. Haemophilia. 2010;16(1):e190-201.

4. Escuriola-Ettingshausen C, Kreuz W. Recombinant vs. plasma-derived products, especially those with intact VWF, regarding inhibitor development. Haemophilia. 2006;12(Suppl 6):102-106.

5. Goudemand J, Rothschild C, Demiguel V, et al. Influence of the type of factor VIII concentrate on the incidence of factor VIII inhibitors in previously untreated patients with severe hemophilia A. Blood. 2006;107(1):46-51.

6. Chalmers EA, Brown SA, Keeling D, et al. Early factor VIII exposure and subsequent inhibitor development in children with severe haemophilia A. Haemophilia. 2007:13(2):149-155.

7. Iorio A, Halimeh S, Holzhauer S, et al. Rate of inhibitor development in previously untreated hemophilia A patients treated with plasma-derived or recombinant factor VIII concentrates: a systematic review. J Thromb Haemost. 2010;8(6):1256-1265.

8. Franchini M, Tagliaferri A, Mengoli C, Cruciani M. Cumulative inhibitor incidence in previously untreated patients with severe hemophilia A treated with plasma-derived versus recombinant factor VIII concentrates: a critical systematic review. Crit Rev Oncol Hematol. 2012;81(1):82-93

9. Marcucci M, Mancuso ME, Santagostino E, et al. Type and intensity of FVIII exposure on inhibitor development in PUPs with haemophilia A. A patient-level meta-analysis. Thromb Haemost. 2015;113(5):958-967.
10. Gouw SC, van der Bom JG, Ljung R, et al. Factor VIII products and inhibitor develop ment in severe hemophilia A. N Engl J Med. 2013;368(3):231-239.

11. Calvez T, Chambost H, ClaeyssensDonadel S, et al. Recombinant factor VIII products and inhibitor development in previously untreated boys with severe hemophilia A. Blood. 2014;124(23):3398-3408.

12. Collins PW, Palmer BP, Chalmers EA, et al. Factor VIII brand and the incidence of factor VIII inhibitors in previously untreated UK children with severe hemophilia A, 20002011. Blood. 2014;124(23):3389-3397.

13. Kessler CM, Iorio A. The Rodin (Research Of Determinants of INhibitor Development among PUPs with haemophilia) study: the clinical conundrum from the perspective of haemophilia treaters. Haemophilia. 2013;19(3):351-354.

14. van der Bom JG, Gouw SC, Rosendaal FR. Second-generation recombinant factor VIII and inhibitor risk: interpretation of RODIN study findings and implications for patients with haemophilia A. Haemophilia. 2014;20(2):e171-174

15. Berntorp E, Iorio A. Reflections on the FranceCoag report on inhibitory antibodies to factor VIII in patients with severe hemophilia A. Blood. 2015;125(24):3816-3817.

16. Calvez T, Chambost H, Lutz P, Rothschild C, Goudemand J. Response: confounding by indication is unlikely to explain the higher inhibitor incidence in boys treated with a recombinant FVIII product Blood. 2015;125(24):3817-3819.

17. Mathew P, Dinter H, Church N, Humphries TJ, Kulkarni R. Inhibitors in haemophilia A a perspective on clotting factor products as a potential contributing factor. Haemophilia. 2016;22(3):334-341.

18. Peyvandi F, Mannucci PM, Garagiola I, et al. A randomized trial of factor VIII and neutralizing antibodies in hemophilia A. N Engl J Med. 2016;374(21):2054-2064.

19. Peyvandi F, Mannucci PM, Palla R, Rosendaal FR. SIPPET: methodology, analysis and generalizability. Haemophilia. 2017;23(3):353-361
20. European Medicines Agency. Consensus points - Workshop on Haemophilia Registries [Internet]. 2015 [cited Mar 29, 2016]; Available from: http://www.ema. europa.eu/docs/en_GB/document_library/ Other/2015/08/WC500191378.pdf.

21. van den Berg HM, Peyvandi F. Assessment of clotting factor concentrates - pivotal studies and long-term requirements. Semin Thromb Hemost. 2015;41(8):855-859.

22. Carcao M, Re W, Ewenstein B. The role of previously untreated patient studies in understanding the development of FVIII inhibitors. Haemophilia. 2016;22(1):22-31.

23. Calvez T, Biou M, Costagliola D, et al. The French haemophilia cohort: rationale and organization of a long-term national pharmacosurveillance system. Haemophilia. 2001;7(1):82-88

24. Stonebraker JS, Bolton-Maggs PHB, Soucie JM, Walker I, Brooker M. A study of variations in the reported haemophilia A prevalence around the world. Haemophilia. 2010;16(1):20-32.

25. Meijer P, Verbruggen B. The between-laboratory variation of factor VIII inhibitor testing: the experience of the external quality assessment program of the ECAT foundation. Semin Thromb Hemost. 2009;35(8): 786-793.

26. Chtourou S, Porte P, Nogré M, et al. A solvent/detergent-treated and 15-nm filtered factor VIII: a new safety standard for plasma-derived coagulation factor concentrates. Vox Sang. 2007:92(4):327-337.

27. Parti R, Schoppmann A, Lee H, Yang L. Stability of lyophilized and reconstituted plasma/albumin-free recombinant human factor VIII (ADVATE rAHF-PFM) Haemophilia. 2005;11(5):492-496.

28. Jiang R, Monroe T, McRogers R, Larson PJ Manufacturing challenges in the commercial production of recombinant coagulation factor VIII. Haemophilia. 2002;8 (Suppl 2):1-5

29. Austin PC. The relative ability of different propensity score methods to balance measured covariates between treated and untreated subjects in observational studies. 
